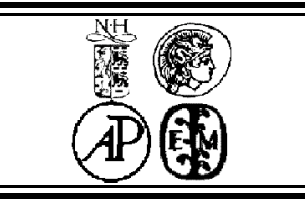

ELSEVIER

\title{
Static and dynamic properties of three-dimensional dot-type magnonic crystals
}

\author{
Artur Maksymov ${ }^{\mathrm{a}, \mathrm{c}^{*}}$, Leonard Spinu ${ }^{\mathrm{a}, \mathrm{b}}$ \\ a Advanced Material Research Institute, University of New Orleans, LA 70148, USA. \\ ${ }^{\mathrm{b}}$ Department of Physics, University of New Orleans, New Orleans, LA 70148, USA. \\ ${ }^{\mathrm{c}}$ Department of General Physics, Chernivtsi National University, Chernivtsi 58012, Ukraine. \\ Elsevier use only: Received date here; revised date here; accepted date here
}

\begin{abstract}
The static and dynamic magnetization of three-dimensional magnonic metamaterials has been investigated. By numerical means it was analyzed the impact of space dimensionality on the properties of magnonic crystal with unit cell consisting of four dots. It is find out the possibility of multi-vortex core formation which is related to the increasing of the crystal height by three-dimensional periodicity of single crystal layer. Additionally is provided the analysis of ferromagnetic resonance phenomenon for two-dimensional and three-dimensional structures. For the unsaturated magnetization of three-dimensional crystal the several pronounced resonance frequencies were detected.
\end{abstract}

(C) 2013 Elsevier Science. All rights reserved

Keywords: magnonic crystal, spin waves, vortex behavior.

\section{Introduction}

The existence and propagation of spin waves in magnetic media with forbidden energy gap raised an extensive scientific interest in so called magnonic crystal (MC) - a magnetic counterpart of photonic crystal [1-4]. The concepts of artificial crystals or metamaterials developed for electromagnetic waves in the optical and microwave frequency regime applied to spin waves show great advantages for their theoretical description and experimental studies. Magnonic metamaterials or magnonic crystals are artificial assembly of "man-made" building blocks with tailored properties via their geometrical shaping and compositional modulation [3]. The possibility to reproduce the properties of magnonic crystals which are similar to the ones observed in photonic crystals, but controlled by external applied magnetic fields makes these materials very interesting from point of view of their practical applications. Among the prospective materials for future electronics and logic applications, magnonic crystals have a special role related to the possibility of using the electron's spin for information storage and implementation of spin wave based logics for information processing [4].

The predetermination of magnonic crystals properties by external magnetic fields and their geometrical configurations lead to various studies during last period [5-8]. The previous theoretical and experimental studies of magnonic crystals were focused mainly on one-dimensional (1D) and twodimensional (2D) MCs, i.e., magnetic structures with a periodic modulation of magnetic properties in one or two dimensions, respectively. Thus, micromagnetic simulation of MCs were carried by considering systems based on a unit cell of a certain configuration and periodically repeated in one or two dimensions using periodic boundary conditions. [911]. For reasons related to difficulties of performing large scale micromagnetic siumlations, and experimental fabrication of three-dimensional magnonic crystals, systematic studies of 3D MCs are lacking.

In this work we present a systematic study of static and dynamic magnetic properties of dot-based 3D MCs and compare with the results obtained for corresponding $1 \mathrm{D}$ and 2D systems. The study was done on Permalloy dots arranged in a square unit cell. The theoretical description was provided within classical phenomenological approach of magnetization dynamics based on Landau-LifshitszGilbert (LLG) equation. The micromagnetic simulations were carried out by using the opensource Graphics Processing Unit - GPU accelerated micromagnetic code mumax 3 [12]. The size of the studied MC was obtained by considering periodic

*Corresponding author: e-mail: maxyartur@gmail.com (Artur Maksymov) 
boundary conditions using the macro-geometry approach [13] where a finite number of periodic copies are taken into account in each direction. The static magnetic properties were analyzed by following the system's spin configuration during major hysteresis loop. Frequency and magnetic field dependent dynamic susceptibility simulations were considered to reveal, the dynamic properties of 3D MCs.

\section{Micromagnetic Simulations}

There were previous reports of studies on certain configurations of dot-based MCs [6, 14-18]. In the present work in order to have a better understanding of the influence of the dimensionality of the dots' arrangement on the properties of $\mathrm{MCs}$, we considered a systematic study starting from a single dot of diameter $D=500 \mathrm{~nm}$ and height $h=20 \mathrm{~nm}$. Then, a single square unit cell of four dots with an edge to edge distance $l=500 \mathrm{~nm}$ was considered, and one continued with different sizes of 2D dots arrangements obtained using macro geometry periodic boundary conditions. The 3D MC was built by repeating the $2 \mathrm{D}$ dots arrangements using macrogeometry periodic boundary conditions with a interlayer gap of $g=20 \mathrm{~nm}$.

The description of magnetization dynamics in the system is provided in the framework of LandauLifshitz-Gilbert equation [19-21], including the energy dissipation term.

$$
\frac{\partial \vec{M}(\vec{r}, t)}{\partial t}=-\gamma \mu_{0} \vec{M}(\vec{r}, t) \times \vec{H}_{e f f}(\vec{r}, t)+\vec{R} .
$$

Here, the gyromagnetic ratio is $\gamma=g \mu_{B} / \hbar$ where $\mu_{B}$ is the Bohr magneton, $\hbar$ is the reduced Planck constant and $g$ is so called $g$ - factor depending on atomic quantum numbers. The term $\vec{H}_{e f f}(\vec{r}, t)$ denotes the effective magnetic field acting on the magnetic moment. The last term in equation $\vec{R}$ corresponds to the dissipation effects.

The phenomenological form of Landau- LifshitzGilbert (LLG) equation, which is implemented in used simulation software, is [12]:

$$
\begin{aligned}
& \frac{d \vec{M}(\vec{r}, t)}{d t}=-\frac{\gamma}{1+\alpha^{2}} \vec{M}(\vec{r}, t) \times \vec{H}_{e f f}(\vec{r}, t)- \\
& \quad-\frac{\alpha \gamma}{M_{s}\left(1+\alpha^{2}\right)} \vec{M}(\vec{r}, t) \times\left[\vec{M}(\vec{r}, t) \times \vec{H}_{e f f}(\vec{r}, t)\right]
\end{aligned}
$$

The effective magnetic field $\vec{H}_{e f f}(\vec{r}, t)$, which acts on the local magnetization vector $\vec{M}(\vec{r}, t)$ of the sample besides the uniform and constant applied field $\vec{H}_{0}$, in general, is the sum of several additional components like, exchange field $\vec{H}_{e x}(\vec{r}, t)$, demagnetized field $\vec{H}_{\text {demag }}(\vec{r}, t)$, anisotropy field $\phi_{a n i}(\vec{r})$ temperature field and other

$$
\begin{gathered}
\vec{H}_{e f f}(\vec{r}, t)=\vec{H}_{0}(\vec{r})+\vec{H}_{\text {demag }}(\vec{r}, t)+\vec{H}_{e x}(\vec{r}, t)+ \\
+\phi_{\text {ani }}(\vec{r})+\text { other }
\end{gathered} .
$$

For our simulations the effective field is simplified by considering isotropic material properties, i.e. $\phi_{a n i}(\vec{r})=0$ and neglecting the actions of other fields which comparatively to the action of internal field make small contribution in the magnetization dynamics.

The definition of demagnetized field $\vec{H}_{\text {demag }}(\vec{r}, t)$ for the various shape bodies is still a difficult problem and needs to be considered separately for each particular case due to its dependence on bodies shape.

For the studied model, the demagnetized field accounting for the long-range interaction throughout the complete sample and representing the solution of Maxwell's equations in the magnetostatic approaches is

$$
\vec{H}_{\text {demag }}(\vec{r}, t)=-\frac{1}{4 \pi} \int_{V} \nabla \nabla \frac{1}{\left|\vec{r}-\vec{r}^{\prime}\right|} \vec{M}\left(\vec{r}^{\prime}\right) d \vec{r}^{\prime} .
$$

The spatiotemporal exchange field for the case of magnetically homogeneous media studied here makes a contribution to the effective field (3) in the form of a laplacian of the magnetization

$$
\vec{H}_{e x}(\vec{r}, t)=\frac{2 A}{\mu_{0} M_{s}^{2}} \nabla^{2} \vec{M}(\vec{r}, t),
$$

where $A$ is the exchange stiffness constant, depending on the coordinates for the inhomogeneous media. For our simulations we chose the permaloy material, which is characterized by exchange stiffness constant $A=13 \cdot 10^{-12} \mathrm{~J} / \mathrm{m}$ and saturation magnetization $M_{s}=8 \cdot 10^{5} \mathrm{~A} / \mathrm{m}$.

The properties of studied theoretical model of magnonic crystal were simulated by using open source micromagnetic simulation package mumax3, which provides the numerical solving of LLG equation (2) with effective field (3). The micromagnetic package mumax 3 is based on finite difference (FD) numerical method and is characterized by implementation on GPUs and thus has good parallelization even on the desktop computers with CUDA supported graphic cards. The good review of advantages and performance of mumax3 implemented on GPUs comparatively to numerical simulations on computational processor units (CPU) is reported here [12]. 


\section{Results and Discussions}

\subsection{Static magnetization}

The unit cell of the simulated $\mathrm{MC}$ is formed by four permaloy dots. Applying macro-geometry periodic boundary conditions we studied several MC sizes and the results obtained were compared with those reported in literature [15-18]. In order to understand the effects of inter-dot interaction, system size and dimensionality we focused on a unit cell consisting of four equidistant dots for which several periodic boundary conditions were considered. In Fig. 1 are shown the hysteresis loops obtained for one dot, four dots with a total 2D system size $2000 \mathrm{~nm} \times 2000 \mathrm{~nm}$ and a 2D crystal with a size of $0.5 \mathrm{~mm} \times 0.5 \mathrm{~mm}$ obtained by 125 repetitions of 4dots unit cell in both directions of $X$ and $Y$ axes, i.e. $\operatorname{PBC}(125,125,0)$. One observes that the hysteresis loops for one dot, four dots and the 2D extended crystal are characterized by the existence of a small coercivity and a shape that changes depending on the system as one may expect for Permalloy. However, this is somehow different from what usually it is expected in the case of magnetic dots, where a reversible region and a zero remanent magnetization in the hysteresis loops is observed [22]. The occurrence of the reversible region in the magnetic dots' hysteresis loop is dependent on the thickness of the dots, and for thin dots (or large $D / h$ ratios) as it is the case here, the reversible region is not present. Together with the study of hysteresis loops we are interesting in the domain formation and local orientation of magnetization within the dots of each sample. The profiles of space distribution of static magnetization for several fixed external fields are shown additionally in Fig. 1 for each hysteresis loop. We observed that the magnetization domain microstructure is highly dependent on the geometrical configuration of the simulated system. The simplest magnonic structure where it is possible to observe the emergence and development of a magnetic vortex is a single disc $[17,23,24]$. For the one dot sample the transition between the positive and negative magnetization states is characterized by the appearance of the vortex and its movement along a direction perpendicular to the one along which the magnetic field applied and varied. In the case of four dots sample, the generated vortexes in the left and right pair of dots move from center of the sample to the periphery along the axis perpendicular to the external magnetic field (here this is $X$ axis) as the magnetic field is changed from positive saturation to negative one.

The situation becomes more complex if there is influence of more neighboring dots which was be achieved by applying periodic boundary conditions
(PBC). For the case of $2 \mathrm{D} \mathrm{PBC}$ the vortexes generation within the unit cell of four dots in the same time is observed only in three dots from four and its movement in the left and right pairs of dots occurs from periphery to center for transition from $m_{y}=+1$ to $m_{y}=-1$ and from center to periphery for the reverse transition.
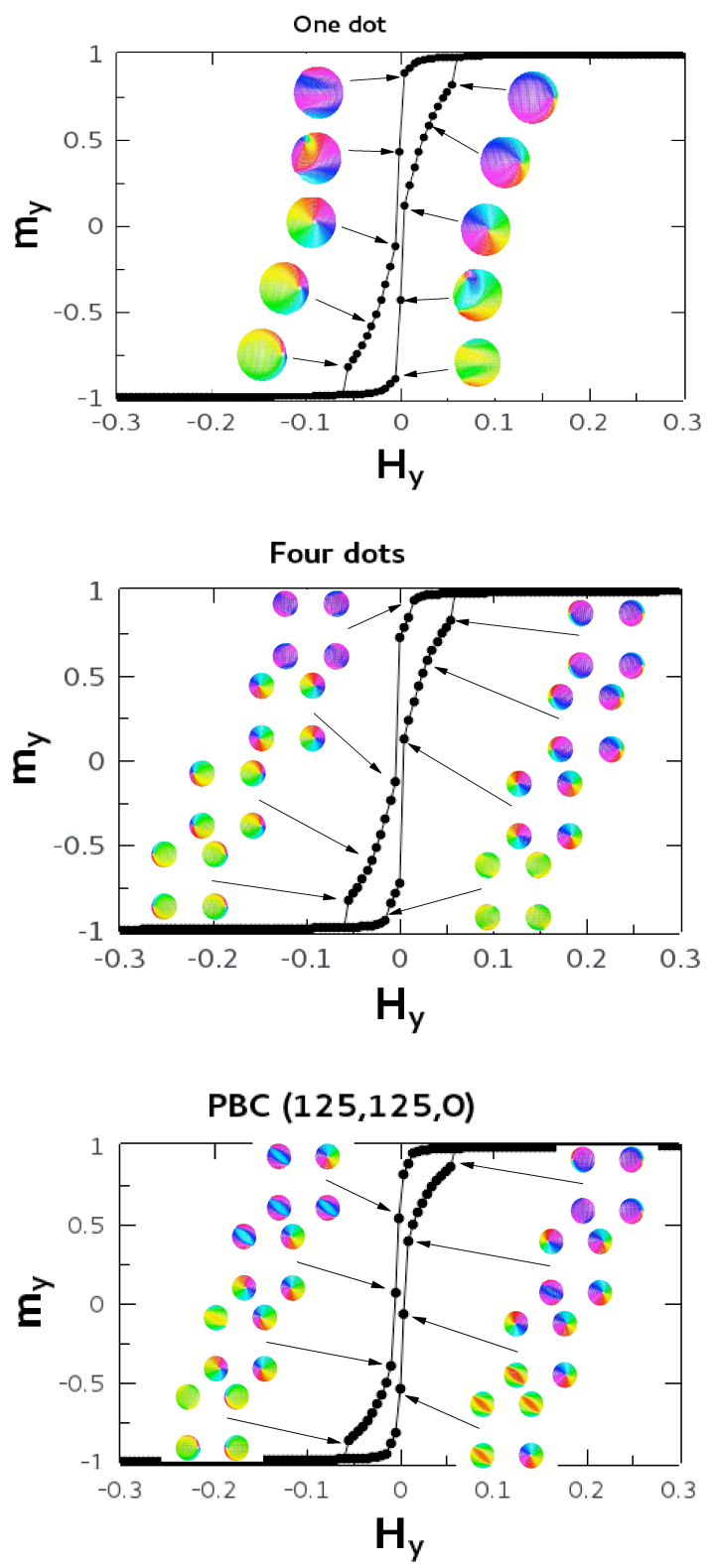

Fig 1. The dependence of hysteresis loop on the geometrical configuration for the single dot, four dots and 2D crystal. The magnetic field is applied along OY (vertical) direction. Additionally the vortex nucleation and annihilation are shown.

The static magnetization behavior of 3D MCs of several heights is obtained by applying periodic boundary condition along $Z$ axis and studying the corresponding hysteresis loops shown in Fig. 2. The hysteresis loops from Fig. 2 have been calculated for $3 \mathrm{D}$ crystals with sizes $0.5 \mathrm{~mm} \times 0.5 \mathrm{~mm} \times 400 \mathrm{~nm}$, 
$0.5 \mathrm{~mm} \times 0.5 \mathrm{~mm} \times 800 \mathrm{~nm}$

and

$0.5 \mathrm{~mm} \times 0.5 \mathrm{~mm} \times 1.6 \mu \mathrm{m}$ which have been obtained by additional periodicity of $2 \mathrm{D}$ structure along $Z$ axis with 5, 10, 20 repetitions respectively.
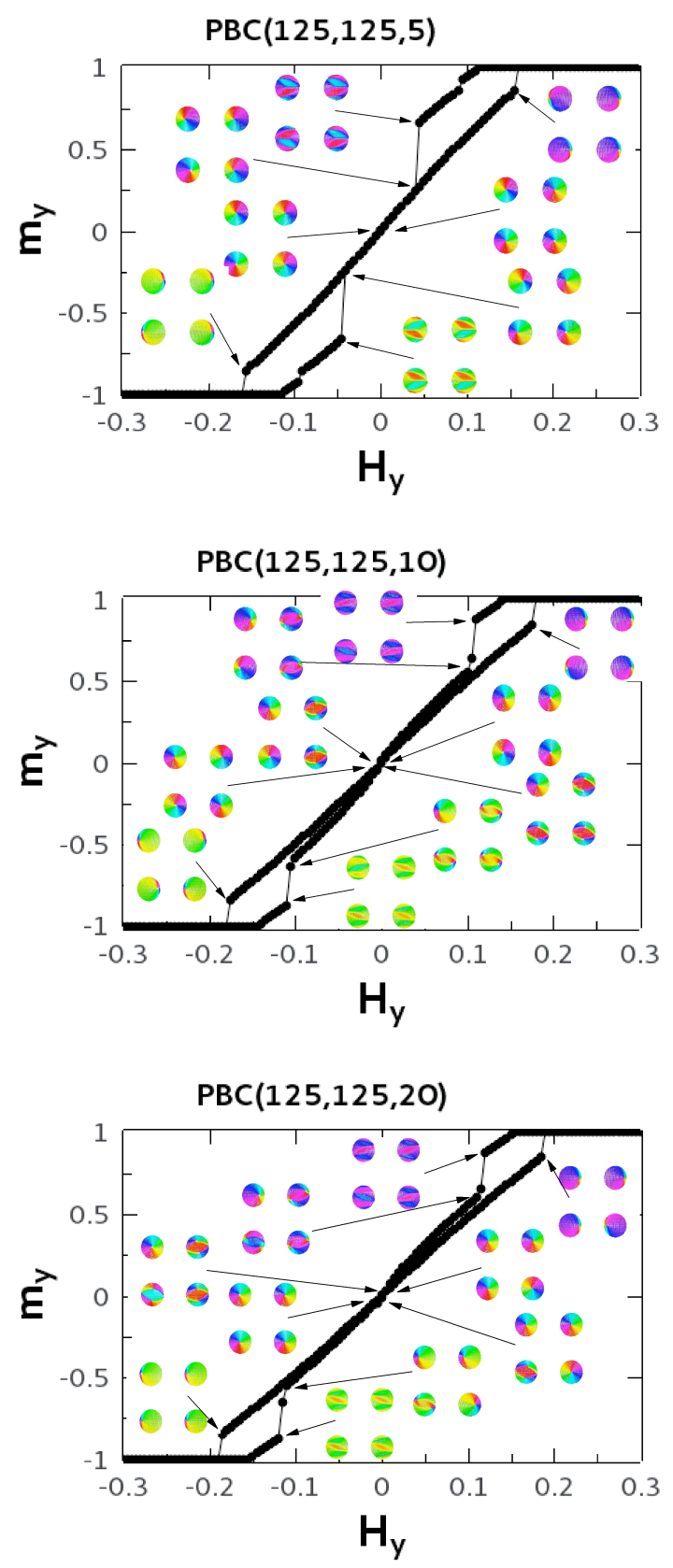

Fig 2. The dependence of hysteresis loop on the height of permaloy magnonic metamaterial. The magnetic field is applied along OY (vertical) direction. For several field values are shown the profiles of magnetization with vortex domain structure.

One observes that the hysteresis loops of $3 \mathrm{D}$ systems are qualitatively different from the ones corresponding to four dots and 2D crystals. For the 3D structures the coercivity becomes zero, and the transition curves is characterized by a double triangle shape, which is typical for the dot with vortex ground state, and forming two bistable regions [16]. These regions correspond to vortex nucleation and annihilation. The hysteresis loops for this case are also characterized by reversible magnetization region, which may be tailored by changing the number of layers in the crystal. The appearance of reversible region is related to weak exchange coupling between the dots from different layers. With increasing crystal size on $Z$ direction, the inclination of the transition curves is increasing and the range of these bistable regions show a tendency to decrease which enhance the region of reversible magnetization.
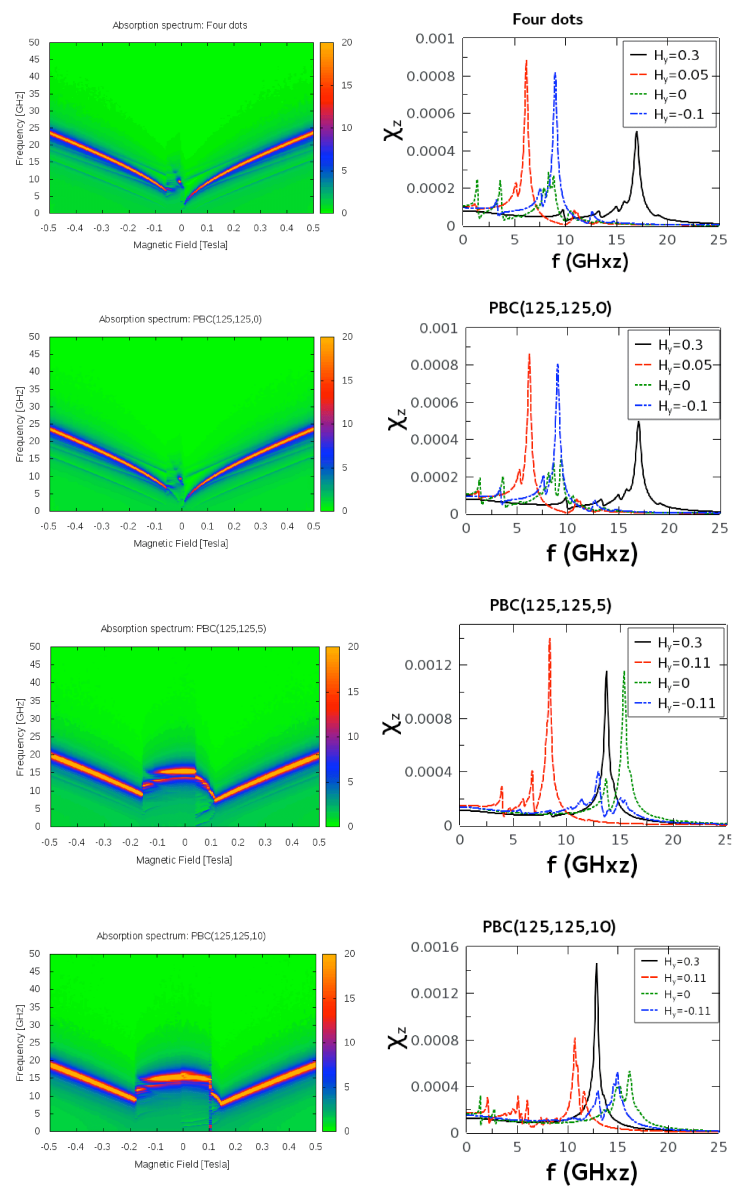

Fig 3. The ferromagnetic resonance obtained from simulations for four dots, 2D array of dots and 3D magnonic crystal with 5 and 10 periodic boundary condition along $Z$.

Besides the changes in the shape of hysteresis loops for the 3D MCs, obtained here by applying additional $\mathrm{PBC}$ on $\mathrm{Z}$ axis, the vortex development in the unit cell of four dots surrounded by the neighbors in all three dimensions is specific too and shows dependence on the size of the crystal along $Z$ axis. First of all we remark that the movement of vortexes in dots may be grouped by top and bottom pairs. For the case with five repetitions along $\mathrm{Z}$ this movement is directed from left to right in the top pair and from center to periphery in the bottom pairs for transition $+1 \rightarrow-1$. For the reverse transition the top and bottom pair is switched. With increasing the number 
of virtual copies along $Z$ axis more complex vortex structure is observed. The vortex nucleation over the dots from unit cell is non-uniform and for some of dots is observed the double vortex core. Moreover, there is asymmetry in the vortex nucleation during transition from +1 to -1 and in reverse direction.

\subsection{Dynamic magnetization}

The studying of magnetic hysteresis loops is one of more comprehensive description of static magnetization, but from point of view of practical applications it is useful to have information about dynamics of magnetization. The magnetization dynamics is a very powerful technique providing the description of phenomena related to the existence and propagation of spin waves in magnetic materials.

Here, we carried out the studying of the ferromagnetic resonance (FMR) phenomenon for the case of four dots, the $2 \mathrm{D}$ and $3 \mathrm{D}$ samples obtained by applying the PBC along corresponding axes. These results are shown in Fig. 3 as dependence of the resonance frequencies corresponding to maximal absorption on the applied external static field. On the right side panels are presented the susceptibility dependencies for several fixed field where the absorption peaks can be observed in more details.

The FMR absorption spectra shown in Fig. 3 have been obtained from mumax 3 simulations by analysis of susceptibility at each value of the DC field oriented along $Y$ axis with the AC field applied along $Z$ axis perpendicular to the plane of the dots. The applied DC magnetic field was swept from $0.5 \mathrm{~T}$ to $-0.5 \mathrm{~T}$ using a field step of $0.005 \mathrm{~T}$, magnetic field range which corresponds to decreasing branch of hysteresis loop. The dynamic excitation was realized by applying an exponential pulse, $\vec{h}_{\text {pulse }}(t)=\vec{H}_{0} \exp \left[-\left(\frac{t-T_{\text {quiet }}}{T_{\text {pulse }}}\right)\right]$ with amplitude of $H_{0}=0.001 \mathrm{~T}$, duration of $T_{\text {pulse }}=40 \mathrm{ps}$ and quiescent time of $T_{\text {quiet }}=500 \mathrm{ps}$ and monitor the time dependence of the magnetization $\vec{M}(t)$. A discrete Fourier transform (DFT) of the time domain data allowed us to obtain the frequency domain field and magnetization data $\tilde{\vec{M}}(\omega)$ and obtain the tensor of complex dynamic magnetic susceptibility

$$
\tilde{\chi}_{i j}(\omega)=\frac{\tilde{M}_{i}(\omega)}{\tilde{h}_{j}}
$$

The Landau-Lifshitz damping constant used in simulations is $\alpha=0.01$. The relative value of dynamic magnetization was stored every 10 ps, which limits the maximum frequency to $50 \mathrm{GHz}$. The simulation time of the experiment is $20 \mathrm{~ns}$. For stored dynamic magnetization by applying DFT was obtained the Fourier images of each magnetization component with real and imaginary part in frequency space.

The obtained FMR spectra show, that the number and composition of resonant modes for four dots and 2D crystal slightly differ. The main difference is observed in unsaturated region and, as is seen, the geometrical configuration of the crystals influences rather on the domains structure and their dynamics. The absorption spectra at zero field are almost the same excepting the main peak at $9 \mathrm{GHz}$, which for $2 \mathrm{D}$ crystal is obtained as two very close peaks.

The FMR images of 3D structures are characterized by larger unsaturated region, within of what the absorption lines are clearly visible. In the result of susceptibility analysis of the saturated area of 3D sample, corresponding to high applied field, only one peak is determined. This peak becomes more pronounced with increasing periodicity along $Z$ axis. Instead of this, the multiple peaks, with better distinctions comparatively to four dots and 2D structure are observed in unsaturated region. For $3 \mathrm{D}$ crystal with 5 layers this region is between $H_{y}=-0.16 \mathrm{~T}$ and $H_{y}=0.16 \mathrm{~T}$, whereas for the sample with 10 layers this region is from $H_{y}=-0.18 \mathrm{~T}$ to $H_{y}=0.18 \mathrm{~T}$.

The analysis of FMR spectra of $2 \mathrm{D}$ and $3 \mathrm{D}$ structures shows the common region with linear dependence of resonance frequencies on applied magnetic field. The region of linear dependence is observed for high values of magnetic fields and corresponds to saturated magnetization of decreasing branch of hysteresis loop. For the case of four dots and 2D crystal the region of unsaturated magnetization is narrower than the one for $3 \mathrm{D}$ crystal and is hard to distinct clearly the resonance frequencies and in general to judge about occurring absorption. For 3D crystal the unsaturated region is wider and occurring absorption processes are clearly visible. The region of hysteresis loop where magnetization decreases gradual and linearly is characterized by two clearly distinguishable resonant frequencies, which are constant on field variation. The increasing of crystal size along $Z$ axis leads to wider region of constant resonant frequencies.

\section{Conclusions}

The static and dynamic magnetization of twodimensional and three-dimensional magnonic crystals were investigated in the framework of macroscopic classical phenomenological model. It was studied the hysteresis loops for four dots, two-dimensional and three-dimensional magnonic crystals obtained by applying periodic boundary condition following macro-geometry approach. It is find out that 
additional periodic boundary condition on $\mathrm{Z}$ axis changes the shape of hysteresis loops for threedimensional materials, and leads to appearance of reversible region.

For three-dimensional crystal the vortex development in the unit cell of four dots is characterized by specific movement during field variation, also depending on the crystal size along $\mathrm{Z}$ axis. The increasing number of multilayers in the crystal leads to the double vortex-core behavior, which is observed for some dots from the unit cell.

For several crystal size and dimensionality the absorption of magnetic field has been modeled and the corresponding ferromagnetic resonance images have been obtained. It was observed that for threedimensional crystals there are wide region of unsaturated magnetization in the low-field region with several possible resonant frequencies, whereas the region with high-field strength is characterized by single absorption frequency.

\section{Acknowledgments}

The work at University of New Orleans was supported by the National Science Foundation under the NSF EPSCoR Cooperative Agreement No. EPS1003897 with additional support from the Louisiana Board of Regents. Portions of this research were conducted with high performance computational resources provided by the Louisiana Optical Network Initiative (http://www.loni.org).

\section{References}

[1] E. Yablonovitch, T. J. Gmitter, and K. M. Leung, Phys. Rev. Lett. 67 (1991) 2295

[2] M. Krawczyk, and H. Puszkarski, Phys. Rev. B 77 (2008) 054437.

[3] M. Krawczyk, and D. Grundler, J. Phys.: Condens. Matter 26 (2014) 123202.
[4] B. Lenk, H. Ulrichs, F. Garbs, and M. Münzenberg, Phys. Rep. 507 (2011) 107.

[5] S.O. Demokritov, A.N. Slavin (eds.), Magnonics: From Fundamentals to Applications, Topics in Applied Physics 125, Springer-Verlag, Berlin, 2013.

[6] A. O. Adeyey, and N. Singh, Large area patterned magnetic Nanostructures, J. Phys. D: Appl. Phys. 41 (2008) 153001.

[7] V. V. Kruglyak, S. O. Demokritov, and D. Grundler, J. Phys. D: Appl. Phys. 43 (2010) 264001.

[8] H. Yu, G. Duerr, R. Huber, M. Bahr, T. Schwarze, F. Brandl, and D. Grundler, Nat. Commun.4 (2013) 2702.

[9] F. Giesen, J. Podbielski, and D. Grundler, Phys. Rev. B 76 (2007) 014431.

[10] S. Neusser, B. Botters, M. Becherer, D. Schmitt-Landsiedel, and D. Grundler, Appl. Phys. Lett. 93 (2008) 122501.

[11] J. Topp, D. Heitmann, M. P. Kostylev, and D. Grundler, Phys. Rev. Lett. 104 (2010) 207205.

[12] A. Vansteenkiste, J. Leliaert, M. Dvornik, M. Helsen, F. Garcia-Sanchez, and B. Van Waeyenberge, AIP Adv. 4 (2014) 107133.

[13] H. Fangohr,G. Bordignon, M. Franchin, A. Knitte, P.A. J. de Groot, and T. Fischbacher, J. Appl. Phys. 105 (2009) 07D529.

[14] E. K. Semenova, F. Montoncello, S. Tacchi, G. Dürr, E. Sirotkin, E. Ahmad, M. Madami, G. Gubbiotti, S. Neusser, D. Grundler, F. Y. Ogrin, R. J. Hicken, V. V. Kruglyak, D. V. Berkov, N. L. Gorn, and L. Giovannini, Phys. Rev. B 87 (2013) 174432.

[15] B. K. Mahato, B. Rana, D. Kumar, S. Barman, S. Sugimoto, Y. Otani, and A. Barman, Appl. Phys. Lett. 105 (2014) 012406.

[16] X. M. Liu, J. Ding, G. N. Kakazei, and A. O. Adeyeye, Appl. Phys. Lett. 103 (2013) 062401.

[17] K. Yu. Guslienko, J. Nanosci. Nanotechno. 8 (2008) 2745

[18] O. Dmytriev, V. V. Kruglyak, M. Franchin, H. Frangohr, L. Giovannini, and F. Montoncello, Phys. Rev. B 87 (2013) 174422.

[19] A. G. Gurevich, G. A. Melkov, Magnetization Oscillations and Waves, CRC Press, Boca Raton, 1996, $464 \mathrm{p}$.

[20] D. D. Stancil, and A. Prabhakar, Spin Waves: Theory and Applications, Springer Science+Business Media, New York, 2009, [21] M. Krawczyk, and H. Puszkarski, J. Appl. Phys. 100 (2006) 073905 .

[22] G. Gubbiotti, G. Carlotti, F. Nizzoli, R. Zivieri, T. Okuno, and T. Shinjo, IEEE Trans. Magn. 38 (2002) 2532.

[23] R. I. Joseph, and E. Schlömann, J. Appl. Phys. 36 (1965) 1579.

[24] S.-B. Choe, Y. Acremann, A. Scholl, A. Bauer, A. Doran, J. Stöhr, H. A. Padmore, Science 304 (2004) 420. 